\title{
Growth Hormone Deficiency: an unusual presentation of Floating Harbor Syndrome
}

\author{
Assimina Galli-Tsinopoulou, ${ }^{1}$ Ioannis Kyrgios, ${ }^{1}$ Eleftheria Emmanouilidou, ${ }^{1}$ \\ Ioanna Maggana, ${ }^{1}$ Eleni Kotanidou, ${ }^{1}$ Paraskevi Kokka, ${ }^{1}$ Charilaos Stylianou ${ }^{1}$
}

${ }^{1}$ Fourth Department of Pediatrics, Medical School, Aristotle University of Thessaloniki, Papageorgiou General Hospital Thessaloniki, Greece

\begin{abstract}
Floating-Harbor Syndrome (FHS) is a very rare condition of unknown etiology characterized by short stature, delayed bone age, characteristic facial features, delayed language skills and usually normal motor development. This syndrome has only once been associated with growth hormone deficiency and precocious puberty in the same patient. We describe a 5/12 year-old girl with the typical features of FHS in whom growth hormone deficiency was diagnosed and two years later central precocious puberty was noted. The patient showed a good response to human recombinant growth hormone as well as gonadotropin releasing hormone analogue treatment.
\end{abstract}

Key words: Floating harbor syndrome, GnRH $\alpha$, Growth hormone deficiency, Precocious puberty, Short stature

\section{INTRODUCTION}

Floating-Harbor Syndrome (FHS; OMIM 136140) is a very rare condition of unknown etiology characterized by short stature, delayed bone age, delayed language skills and distinct facial features such as triangular face, microcephaly, wide mouth with downturned corners, low-set and posteriorly rotated ears, deep-set eyes, long eyelashes, bulbous nose, wide and low-hanging collumella and short neck. Our

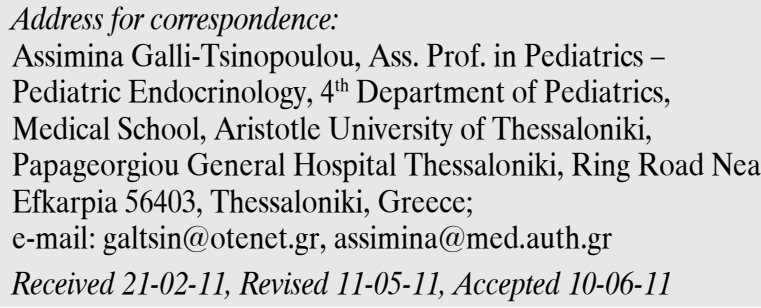

literature search through the PUBMED database revealed that over 50 patients with FHS have so far been described, although the diagnosis of several of these cases was not definitely confirmed. FHS was first described in 1973 by Pelletier and Feingold at the Boston Floating Hospital and in 1975 by Leisti et al. at the Harbor General Hospital. ${ }^{1,2}$ The major and other clinical manifestations of FHS were very recently reviewed in a series of 10 patients. ${ }^{3}$ Growth retardation, which is a major clinical manifestation of FHS, still remains unexplained and requires further elucidation. We report a unique case of a $5^{4} / 12$ yearold girl presenting the typical FHS characteristics, who additionally exhibited growth hormone $(\mathrm{GH})$ deficiency and central precocious puberty. The cause, diagnosis and treatment options of these extremely rare features of FHS are also discussed. 


\section{CASE REPORT}

A $54 / 12$ year-old girl with distinct facial features and failure to thrive since birth was admitted to our department for diagnostic evaluation. She was born through a vaginal delivery after an uneventful fullterm pregnancy. The birth weight was $2.7 \mathrm{~kg}\left(10-25^{\text {th }}\right.$ percentile $)$, the length $50 \mathrm{~cm}\left(50^{\text {th }}\right.$ percentile $)$ and the head circumference $33 \mathrm{~cm}\left(10-25^{\text {th }}\right.$ percentile $)$. She is the second child of non-consanguineous healthy parents of Greek origin (mother 36, father 50 years old). The mother's height was $163 \mathrm{~cm}$ and the father's 165 $\mathrm{cm}$ (target height: $157 \pm 4.5 \mathrm{~cm}$ ). Her sister, $9^{3} / 12$ years, has normal growth $\left(25^{\text {th }}\right.$ percentile $)$ and psychomotor development and lacks dysmorphic features. The rest of her family history was unremarkable.

At the initial presentation at $5 \frac{4}{12}$ years, the diagnosis of FHS was made on the basis of specific clinical features (Figure 1). The physical examination revealed height $99 \mathrm{~cm}(-3.84$ SDS $)$, weight 19 $\mathrm{kg}\left(10-25^{\text {th }}\right.$ percentile) and head circumference 46 $\mathrm{cm}\left(<3^{\text {rd }}\right.$ percentile $)$. The growth velocity was 1.5 $\mathrm{cm} /$ year. The dysmorphic characteristics (Figure 1) included small triangular face with deep-set eyes and long eyelashes, a large nose, low-set ears, wide mouth, short philtrum, high-arch palate, dental abnormalities (supernumerary upper incisor, tooth folding and malocclusion) as well as hand anomalies (clinodactyly of the fifth finger, distal clubbing of fingers). At the chronological age of $5 / 12$ years the bone age was 3 years. The metacarpophalangeal pattern profile (MCPP) analysis revealed mild to moderate shortness of all phalanges (metacarpals, proximal, middle and distal). Evaluation of psychomotor development disclosed severely delayed language skills (delayed motor speech production, hypernasality and restricted vocabulary). Slightly delayed personal adaptive skills and fine motor development were also detected, while gross motor development was appropriate for her chronological age. She also had mild mental subnormality (Goodenough-Harris Drawing Test, IQ: 65). Persistent constipation was also reported; however, laboratory investigation did not reveal any pertinent pathological findings. Celiac disease was ruled out since anti-gliadin, anti-endomysium and anti-transglutaminase antibodies were absent. Results of blood biochemical and urine studies were within the normal range. Thyroid and adrenal tests were found

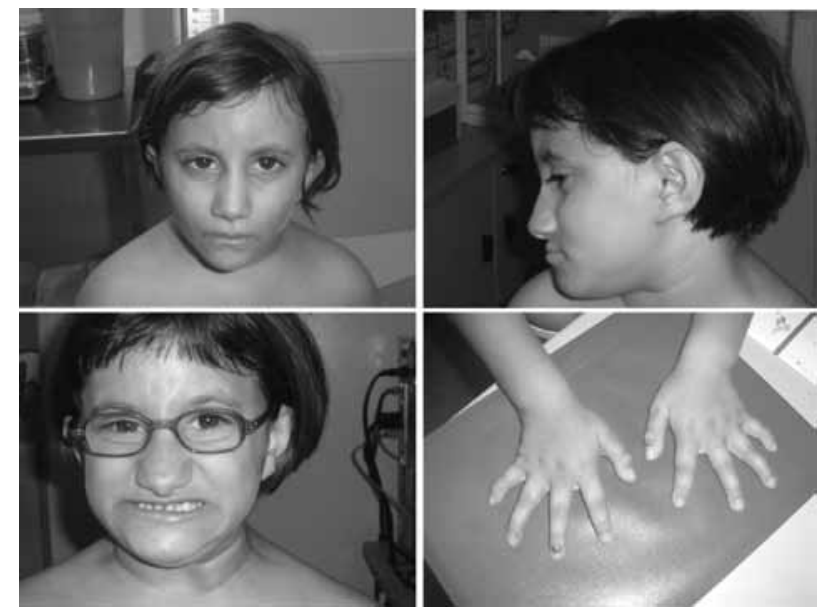

Figure 1. Clinical features of Floating Harbor Syndrome in our patient. Small triangular face, deep-set eyes, long eyelashes, low-set and posteriorly rotated ears and short neck, broad mouth with downturned corners, dental abnormalities, bulbous nose, wide and low-hanging collumella and short philtrum, clubbed fingers and fifth finger clinodactyly.

to be normal as well. GH secretion was evaluated by two provocative tests (glucagon and clonidine) and GH deficiency was detected (peak 5.3 and $5.62 \mu \mathrm{g} / \mathrm{l}$, respectively, normal values $>10 \mu \mathrm{g} / \mathrm{L})$. Furthermore, serum insulin-like growth factor 1 levels were low (36 $\mu \mathrm{g} / \mathrm{l}$ ) for her age and gender (normal range in girls 6-8 years: 26-320 $\mu \mathrm{g} / \mathrm{l}$ ). Hence, recombinant human $\mathrm{GH}$ replacement therapy was initiated $(0.035 \mathrm{mg} / \mathrm{kg} /$ day). In our case, it is noteworthy that during previous evaluation of GH secretion (after glucagon administration), performed at the age of $2^{8} / 12$ years, a normal result had been obtained (peak $21.2 \mu \mathrm{g} / \mathrm{l}$ ). Electoencephalogram, magnetic resonance imaging (MRI) of the brain as well as ophthalmological examination, echocardiography, audiogram and ultrasound of the kidneys were normal. Turner syndrome was excluded by a normal chromosome analysis (karyotype 46, XX). Unfortunately, a banding resolution of chromosomal analysis was not performed.

At the age of $7^{1} / 12$ years, 21 months following GH initiation, an improvement in height was noted [112.5 $\mathrm{cm},(-2.59 \mathrm{SDS})]$, the growth velocity being $8 \mathrm{~cm} /$ year (Figure 2), while her bone age was consistent with her chronological age (7 years). Six months later, at $77 / 12$ years, clinical evaluation showed: height $117 \mathrm{~cm}$ $(-2.22 \mathrm{SDS})$, weight $28.5 \mathrm{~kg}$, growth velocity $9 \mathrm{~cm} /$ year and bone age 8 years. At this time, the patient 


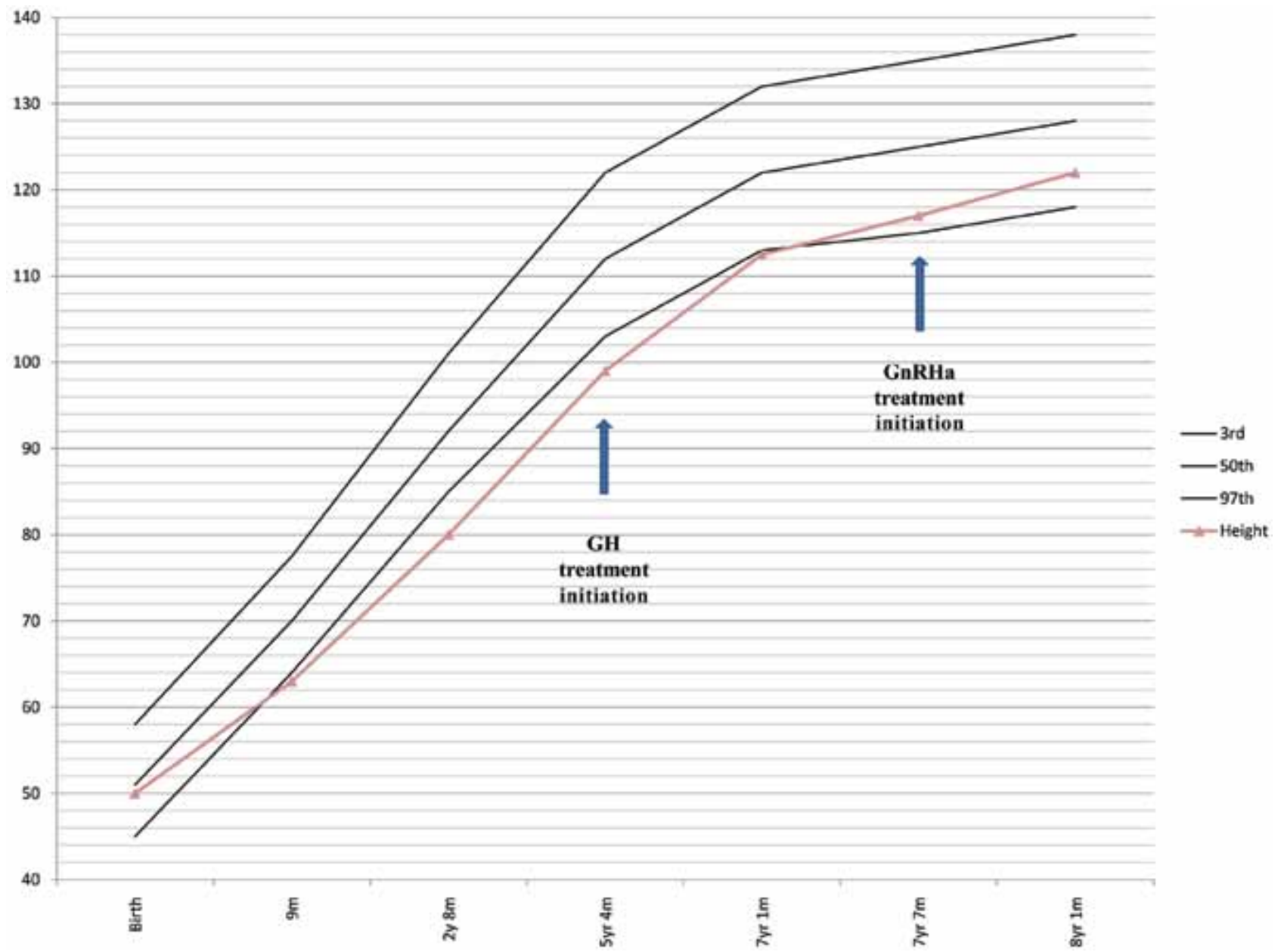

Figure 2. Growth curve of our patient (height in $\mathrm{cm}$ ). Arrows show the age at rhGH and GnRHa initiation.

presented clinical findings of puberty (Tanner stage II breast development and Tanner stage II pubic hair). She had pubertal serum estradiol levels as well as pubertal LH and FSH responses to gonadotropinreleasing hormone $(\mathrm{GnRH})(\mathrm{LH} / \mathrm{FSH}$ ratio $>1)$. Pelvic ultrasonography showed increased uterine volume [3.7 $\mathrm{ml}$ (normal values for age $1.81 \pm 0.44 \mathrm{ml}$ )] with a thickened endometrium $(3 \mathrm{~mm})$; a few follicles (max diameter $6.4 \mathrm{~mm}$ ) were also detected in both ovaries. These findings were consistent with a rapidly progressing pubertal development. On account of the low height at puberty initiation, pubertal arrest was considered necessary. The patient commenced treatment with a $\mathrm{GnRH}$ analogue $(\mathrm{GnRH} \alpha)$ (Triptorelin, ARVEKAP $®, 3.75 \mathrm{mg}$, given intramuscularly, every 28 days).

\section{DISCUSSION}

This is the first patient with FHS reported from Greece. Due to the absence of confirmatory laboratory features in this syndrome, the diagnosis relies on clinical findings and could possibly escape detection. It has been proposed that characteristic facial features should be a prerequisite for a correct diagnosis of FHS, although they become less and less noticeable with advancing age. ${ }^{4}$ Characterization of a patient's nature of speech and language disorder and consideration of growth and bone age have also been recognized as equally important in making a precise FHS diagnosis. ${ }^{3}$ This triad of major features and other findings previously described for FHS were present in our patient and enabled the establishment of diagnosis of FHS. In order to further support our 
clinical diagnosis of FHS, a MCPP was also examined and revealed mild to moderate shortness of all phalanges. This was in accordance with findings previously published in two patients with FHS by Wieczorek et al. ${ }^{5}$ The novel findings in our case is GH deficiency as well as early pubertal onset, conditions that can be resolved with timely treatment if promptly recognized by pediatricians.

The molecular cause of this disorder still remains unknown and most cases described in the literature have been sporadic. Some cases however suggest autosomal dominant ${ }^{6}$ or autosomal recessive inheritance ${ }^{7}$ but no specific gene abnormality has so far been implicated. Thus our patient's normal karyotype was an expected finding. Because of the present limited knowledge, giving genetic counseling about the recurrence risk (after the diagnosis of an affected child in a family) is difficult.

Conditions considered in the differential diagnosis include other dysmorphic syndromes with short stature, mainly Seckel, Velo-Cardio-Facial (VCF) or Shprintzen, 3M (Miller, McKusick, Malvaux) and Rubinstein-Taybi (RT) syndrome. In contrast to our patient, Seckel syndrome is characterized by intrauterine growth retardation and a different facial phenotype (bird-headed). Velo-Cardio-Facial syndrome is characterized by palatal abnormalities, conotruncal heart defect and defective thymic development that results in impaired immune function. However, none of these abnormalities were present in our patient. Moreover, 3M and RT syndromes were excluded due to absence of their unique phenotypical features in our patient (slenderness of the long bones with thin diaphyses and tall spinal bones in $3 \mathrm{M}$ syndrome and peculiar broad thumbs and first toes along with autosomal dominant inheritance in RT syndrome).

Short stature is one of the major criteria for diagnosis of FHS. However, the exact mechanism of shortness is still unclear. Growth hormone deficiency was implicated once as a possible cause, ${ }^{8}$ although the diagnosis of FHS in this patient was not unanimously established. ${ }^{3}$ On the other hand, GH secretion was found to be normal in the vast majority of FHS patients, in whom provocative tests for $\mathrm{GH}$ release had been performed. GH replacement treatment has also been given on a trial basis in FHS patients with no confirmed GH deficiency and the effects of its administration have been considered as either beneficial ${ }^{3,5,8-10}$ or ineffective. ${ }^{11}$ Our patient presented impaired GH response after two provocative tests, indicating GH deficiency apparently of delayed onset. The serum insulin-like growth factor 1 levels were also consistent with this diagnosis. After 21 months of replacement therapy with $\mathrm{GH}$, an increase in growth velocity and height were observed with inappropriate acceleration of bone age. Whether $\mathrm{GH}$ treatment is indeed beneficial in improving final height in FHS remains to be established.

Precocious puberty, if not treated, may also reduce the patient's potential for adult height because of the acceleration of bone-maturing by the increased sex steroid hormones, especially estrogen. Although age at onset of puberty and pubertal development have been reported to be normal in most FHS patients, there have been a few cases with precocious puberty. ${ }^{3,10}$ In these cases as in others,${ }^{12}$ the administration of a GnRH analogue leads to suppression of gonadotropins and gonadal sex steroids secretion, reduced growth rate and arrested bone maturation with potential improvement of final height. A 3-year course of fluoxymesterone therapy in one FHS patient did not bring the height within the normal range. ${ }^{13}$ Our patient is the third FHS patient reported in the literature who exhibited precocious puberty confirmed by clinical, hormonal and sonographical findings and to whom GnRH analogue treatment was administered. Obviously, the effect on final height cannot be predicted.

\section{CONCLUSION}

A GH deficiency has rarely been reported in FHS. Based on current data, evaluation of GH secretion should be performed in FHS patients in order to exclude GH deficiency and should moreover probably be repeated if such investigation showed normal $\mathrm{GH}$ values at an earlier age. Although the cause of growth retardation remains unknown, GH replacement therapy may prove beneficial in these patients.

\section{REFERENCES}

1. Pelletier G, Feingold M, 1973 Case report 1. In: Bergasma D, ed. Syndrome Identification. White Plains, NY: National Foundation-March of Dimes: 8-9. 
2. Leisti J, Hollister DW, Rimoin DL, 1975 The FloatingHarbor syndrome. Birth Defects Orig Artic Ser 11: 305-309.

3. White SM, Morgan A, Da Costa A, et al, 2010 The phenotype of floating-harbor syndrome in 10 patients. Am J Med Genet Part A 152: 821-829.

4. Ala-Mello S, Peippo M, 2004 The first Finnish patient with the floating-harbor syndrome: The follow-up of eight years. Am J Med Genet 130: 317-319.

5. Wieczorek D, Wusthof A, Harms E, Meinecke P, 2001 Floating-Harbor syndrome in two unrelated girls: Mild short stature in one patient and effective growth hormone therapy in the other. Am J Med Genet 104: 47-52.

6. Lacombe D, Patton MA, Elleau C, Battin J, 1995 FloatingHarbor syndrome: Description of a further patient, review of the literature, and suggestion of autosomal dominant inheritance. Eur J Pediatr 154: 658-661.

7. Joan DM, Fryns JP, 2003 Floating-Harbor syndrome in two sisters: Autosomal recessive inheritance or germinal mosaicism? Genet Couns 14: 431-433.

8. Cannavò S, Bartolone L, Lapa D, et al, 2002 Abnormalities of GH secretion in a young girl with Floating-Harbor syndrome. J Endocrinol Invest 25: 58-64.

9. Paluzzi A, Viva LJ, Kalsi P, Mukerji N, Tzerakis N, Patton MA, 2008 Ruptured cerebral aneurysm in a patient with Floating-Harbor syndrome. Clin Dysmorphol 17: 283-285

10. Stagi S, Galluzzi F, Bindi G, et al, 2007 Precocious puberty in a girl with Floating-Harbor syndrome. J Pediatr Endocrinol Metab 20: 1333-1337.

11. Robinson PL, Shohat M, Winter RM, et al, 1988 A unique association of short stature, dysmorphic features, and speech impairment (Floating-Harbor Syndrome). J Pediatr 113: 703-706.

12. Pasquino AM, Pucarelli I, Accardo F, Demiraj V, Segni M, Di Nardo R, 2008 Long-term observation of 87 girls with idiopathic central precocious puberty treated with gonadotropin-releasing hormone analogs: Impact on adult height, body mass index, bone mineral content and reproductive function. J Clin Endocrinol Metab 93: 190-195.

13. Wiltshire E, Wickremesekera A, Dixon J, 2005 FloatingHarbor syndrome complicated by tethered cord: A new association and potential contribution from growth hormone therapy. Am J Med Genet 136: 81-83. 